\title{
Les nouvelles directives médico-éthiques de I'ASSM pour la prise en charge des patientes et patients en fin de vie
}

\author{
M. Salathé, M. Zimmermann-Acklin
}

La publication des directives pour la prise en charge des patientes et patients en fin de vie a eu un écho retentissant. La nouvelle réglementation de l'assistance au suicide a été le point crucial relevé tant dans les médias que dans les quelque 150 prises de position qui sont parvenues au secrétariat général. Les sociétés cantonales de médecine, les sociétés de discipline médicale et les autorités cantonales (médecins cantonaux inclus) ont, pour la plupart, salué l'assouplissement conditionnel de l'assistance au suicide; toutefois quelques médecins ou autres personnes isolées et certains milieux religieux ont émis de vives critiques au sujet de cette nouvelle réglementation.

Egalement les processus décisionnels chez les patients incapables de discernement ont fait l'objet de nombreuses réactions; lors de la révision des directives, la sous-commission a restructuré et clarifié ce passage du texte tout en restant fidèle à son contenu.

Dans ses Directives médico-éthiques sur l'accompagnement médical des patients en fin de vie ou souffrant de troubles cérébraux extrêmes de 1995, l'ASSM avait clairement spécifié que l'assistance au suicide n'était pas un acte médical. Les nouvelles Drectives pour la prise en charge des patientes et patients en fin de vie, qui ont été adoptées par le Sénat et qui n'incluent plus les patients souffrant de troubles cérébraux extrêmes, confirment cette déclaration, mais tiennent également compte de l'évolution des mentalités concernant l'autonomie des patients en assouplissant la réglementation de l'assistance au suicide. Cette nouvelle réglementation définit une ligne de conduite claire, également pour les médecins, dans la pratique du suicide assisté - actuellement environ 0,36\% des cas de décès en Suisse selon l'étude EURELD - qui, aux termes de l'article 115 du Code Pénal, n'est pas punissable.

Les directives précisent clairement que le rôle du médecin auprès de patientes et patients en fin de vie est de «soulager les symptômes et d'accompagner le patient». Sur la base des remarques qui sont parvenues au secrétariat pendant la période de consultation, la précision suivante a été apportée: «Il n'est pas de son devoir de proposer une assistance au suicide, mais, bien au contraire, d'apaiser, autant que possible, les souffrances qui pourraient être à l'origine d'un désir de suicide.»

Toutefois, selon les directives mises en consultation, dans des situations exceptionnelles, le médecin pourrait être confronté à un cas de conscience qui exige de prendre une décision qu'il s'agit de respecter. C'est pourquoi, il est stipulé: «D'une part, l'assistance au suicide ne fait pas partie de l'activité médicale, car elle est contraire aux buts de la médecine. D'autre part, le respect de la volonté du patient est fondamental dans la relation médecin-patient. Un tel dilemme exige une décision morale personnelle du médecin qui doit être respectée en tant que telle.» Ensuite sont énumérées les exigences minimales devant être respectées en cas de décision d'aide au suicide.

La version définitive tient également compte des remarques formulées pendant la période de consultation quant à la pression qu'un patient pourrait exercer sur le médecin pour qu'il lui prête son assistance au suicide. La nouvelle version des directives précise qu'un médecin a, dans tous les cas, le droit de refuser d'assister un suicide. En outre, il est explicitement formulé que les dirigeants peuvent interdire l'assistance au suicide à leurs collaboratrices et collaborateurs; par contre, ils ne peuvent pas les y contraindre. Malgré de nombreuses suggestions concernant l'extension du champ d'application de ces directives aux patientes et patients qui ne sont pas en phase terminale, la sous-commission a décidé de s'en tenir à la version d'origine. Ainsi, il est clairement établi que les présentes directives ne s'adressent pas aux patientes et patients désireux de mettre fin à leurs jours en raison d'une maladie ou d'un handicap grave.

Grâce à une prise en charge palliative complète de ces patients ainsi que des patients en fin de vie, les souffrances à l'origine d'un désir de suicide pourraient être soulagées. 